\title{
In vitro study of the effect of doxorubicin released from EVAL membrane on vesical cancer cells
}

\author{
Chun-Hsu Yao ${ }^{d, *}$, Wen-Yuan Chuang ${ }^{a}$, Yueh-Sheng Chen ${ }^{b}$, Tai-Horng Young ${ }^{c}$ \\ ${ }^{a}$ Institute of Materials science and Engineering, College of Engineering, \\ National Taiwan University, Taipei, Taiwan, ROC \\ ${ }^{\mathrm{b}}$ Institute of Chinese Medical Science, China Medical College, Taichung, Taiwan, ROC \\ ${ }^{\mathrm{c}}$ Institute of Biomedical Engineering, College of Medicine and College of Engineering, National Taiwan University, Taipei, Taiwan, ROC \\ ${ }^{\mathrm{d}}$ Department of Radiological Technology, Chungtai Institute of Health Science and Technology, Taichang, Taiwan, ROC
}

Received 12 July 2000; received in revised form 14 February 2001; accepted 16 February 2001

\begin{abstract}
A mixture of poly(ethylene-co-vinyl alcohol) (EVAL), dimethyl sulfoxide (DMSO) and doxorubicin was precipitated from the 1-octanol to form membranes with particles bonded to each other. The drug release profile from the EVAL membrane was investigated for $96 \mathrm{~h}$. Results showed that the release behavior of doxorubicin was increasing with time and a two-step release behavior, a fast and a slow releasing pattern was revealed. It was also found that the number of cancer cells could be significantly decreased by released doxorubicin from the EVAL membrane. It is concluded that the EVAL copolymer could be an ideal encapsulating material for drugs to treat chronic diseases. (C) 2002 Elsevier Science B.V. All rights reserved.
\end{abstract}

Keywords: EVAL; DMSO; Doxorubicin; Drug release; Cancer cell

\section{Introduction}

Despite the rapid development of medicinal and pharmaceutical chemistry in recent decades, chemotherapy is still a major challenge to the cancer patients. Any potent drug to treat the cancer can be very toxic and often less than $1 \%$ of the injected drug molecules can reach their target cells, whereas the rest may damage healthy cells and tissues [1]. To improve this disadvantage, drug delivery system which can change pharmacokinetics and biodistribution of encapsulated agents, reducing toxic side effects and increasing efficacy of the therapy can be a very useful apparatus.

In preprogrammed drug delivery system, drug reservoir can be prepared by homogeneously dispersing drug particles in a diffusion control of polymer matrix [2]. The drug dispersion in the polymer matrix is accomplished by either blending a therapeutic dose of finely ground drug particles with a liquid polymer, followed by cross-linking of polymer chains, or mixing drug solids with a rubbery polymer at an elevated temperature $[3,4]$. It can also be fabricated by

\footnotetext{
*Corresponding author. Tel.: 886-4-2391647/ext. 4112; fax: 886-4-2396705.

E-mail address: chyao@chtai.ctc.edu.tw (C.-H. Yao).
}

dissolving the drug and the polymer in a common solvent, followed by solvent evaporation at an elevated temperature or under a vacuum condition [5].

As discussing the material membrane used to control the drug release, Loeb and Sourirajan was the first group to develop a method to produce asymmetric membrane [6] and since then, the field of membrane has experienced increasing growth. In 1979, Yamashita et al. utilized poly(ethylene-co-vinyl alcohol) (EVAL) copolymer to produce membranes having improved permeability characteristics for the dialysis of blood [7]. It was found that the EVAL membrane consisted of particles bonded to each other with an average diameter within the ranges between 100 and $1000 \AA$. Further, the EVAL can be also prepared by phase inversion from dimethyl sulfoxide (DMSO) solvent and coagulated in the bath of 1-octanol [8,9]. Young et al. have successfully used this new type of membrane structure for drug delivery that offers a biphasic release behavior lasting for $8 \mathrm{~h} \mathrm{[10].} \mathrm{In} \mathrm{their} \mathrm{study,} \mathrm{the} \mathrm{drug} \mathrm{release} \mathrm{in} \mathrm{the} \mathrm{first}$ step was rapid since the membrane was macroscopically porous and the drug outside the EVAL particles diffused out rapidly. In comparison, the drug release in the second step was slow and prolonged. This is probably because the released drugs in this step were those entrapped inside the particles as the membrane was formed. Therefore, it was 
more difficult for those encapsulated drugs to diffuse out of the membrane and into the surrounding medium.

It has been found in recent studies that doxorubicin is a potent antineoplastic agent, used either as the sole agent or in combination chemotherapy against solid tumors [11-13]. In addition, it is known that the doxorubicin is a hydrophilic molecule and can dissolve in DMSO. In this study, we tried to use the EVAL copolymer dissolved in DMSO as the drug carrier to encapsulate the doxorubicin and to investigate the drug-releasing pattern and the effect of released doxorubicin on vesical cancer cells.

\section{Materials and methods}

\subsection{Membrane preparation}

The membrane material studied in this work was the EVAL copolymer, which was kindly supplied by Kuraray Co. Ltd. (Japan). The EVAL contained ca. $56 \mathrm{~mol} \%$ vinyl alcohol (intrinsic viscosity $=0.87 \mathrm{dl} / \mathrm{g}$, measured $M_{\eta}=$ $56000 \mathrm{~g} / \mathrm{mole}$ ) [14]. EVAL membranes were prepared using the phase inversion method described as follows [15]. An appropriate amount of EVAL was dissolved in DMSO at $60^{\circ} \mathrm{C}$ to form $25 \%$ homogeneous polymer solution. The EVAL solution was kept at $25^{\circ} \mathrm{C}$ for $24 \mathrm{~h}$ and spread using an autocoater on a glass plate to form a thin film with a uniform thickness about $100 \mu \mathrm{m}$. Subsequently, the polymer-coated glass plate was immersed into a coagulation bath with 1-octanol to obtain a membrane called 'R-1'. Similar procedures were used to fabricate the EVAL 'R-2' membrane. First, $2 \mathrm{mg} / \mathrm{ml}$ of doxorubicin (Farmitalia Co. Ltd., Italy) dissolved in DMSO at room temperature was prepared. This mixture was then mixed with the EVAL in a weight ratio of 1:20. After coating and casting procedures of the copolymer, both the R-1 and R-2 membranes were removed from the laminate under a vacuum condition. The morphology of the membranes was then examined using an SEM (JEOL-840A) after it was freeze-dried.

\subsection{Drug release study}

In vitro drug release behavior of the EVAL R-2 membrane was determined using 24-welled tissue culture polystyrene plates (Costar, USA) at $37^{\circ} \mathrm{C}$ filled with $1 \mathrm{ml}$ of de-ionized water. The prepared membranes with $15 \mathrm{~mm}$ in diameter were placed in the tissue culture plates. A Teflon ring of $15 \mathrm{~mm}$ diameter was then placed on each of the tested membranes in the wells to prevent them from floating. At selected intervals of time, $200 \mu \mathrm{l}$ of de-ionized water were removed from the sample well. The concentration of released doxorubicin in de-ionized water was measured homogeneously using an UV spectrophotometer at $233 \mathrm{~nm}$ [16]. After the drug concentration was determined, $200 \mu \mathrm{l}$ of de-ionized fresh water was placed back to the well to keep the same volume of receptor. The behavior of drug release was observed for $96 \mathrm{~h}$. All the data were averages of six independent measurements.

\subsection{Cancer cell culture}

The cancer cell used in this study was the 'T24' vesical cell line. Culture was maintained in a $37^{\circ} \mathrm{C}$ water-jacketed incubator equilibrated with $5 \% \mathrm{CO}_{2}$ and kept at approximately $99 \%$ relative humidity. The cells were routinely grown in RPMI-1640 medium containing 10\% fetal bovine serum (Gibco, USA) and 1\% antibiotic-antimycotic (Gibco, USA) in a $75 \mathrm{~cm}^{2}$ cell culture flask (Costar, USA.). The prepared membranes, sterilized in $70 \%$ alcohol overnight and rinsed extensively with distilled water, were placed in 24-welled tissue culture polystyrene plates. A $15 \mathrm{~mm}$ diameter of sterilized Teflon ring was placed on each of the tested membranes in the wells to prevent them from floating. All the membranes were then washed twice with Dulbecco's PBS. After aspiration of PBS, $1 \mathrm{ml}$ medium of cell suspension at a density of $1 \times 10^{5}$ cells/ml RPMI-1640 medium was placed in each of the well and maintained in a humidified atmosphere with $5 \% \mathrm{CO}_{2}$ at $37^{\circ} \mathrm{C}$.

\subsection{MTT assay}

After cell culturing for 4, 24, 48 and $96 \mathrm{~h}$, the viability of fibroblasts was determined by MTT assay. The method described by Mosmann [17] was modified and used in this study. At each period of culturing time, $100 \mu \mathrm{l}$ of MTT solution (Sigma, USA) were added to each well. After $3 \mathrm{~h}$ incubation at $37^{\circ} \mathrm{C}, 200 \mu \mathrm{l}$ of DMSO were added to dissolve the formazan crystals. The combination solution was then jogged homogeneously using a shaker for $15 \mathrm{~min}$. The optical density of the formazan solution was read on an ELISA plate reader (ELx 800, Bio-Tek) at $570 \mathrm{~nm}$. All experiments were repeated four times, and results are expressed as mean \pm standard deviation. The differences between R-1 and R-2 membranes were evaluated using the Student's $t$-test. Significance was assessed at the $P<0.01$ level of confidence.

\section{Results and discussion}

\subsection{Morphology of membranes}

Macroscopically, both the R-1 and R-2 membranes appeared opaque. As shown in Fig. 1, the structure of R-1 membrane was uniform and EVAL particles (ca. $0.4 \mu \mathrm{m}$ in diameter), which fused with each other, were seen on the membrane surfaces to form an interconnected solid phase. In comparison, the structure of R-2 membrane was similar to that of the R-1 membrane (Fig. 2). The particles on both the R-1 and R-2 membranes did not coalesce completely to form a skin layer. Therefore, there should be no difference 


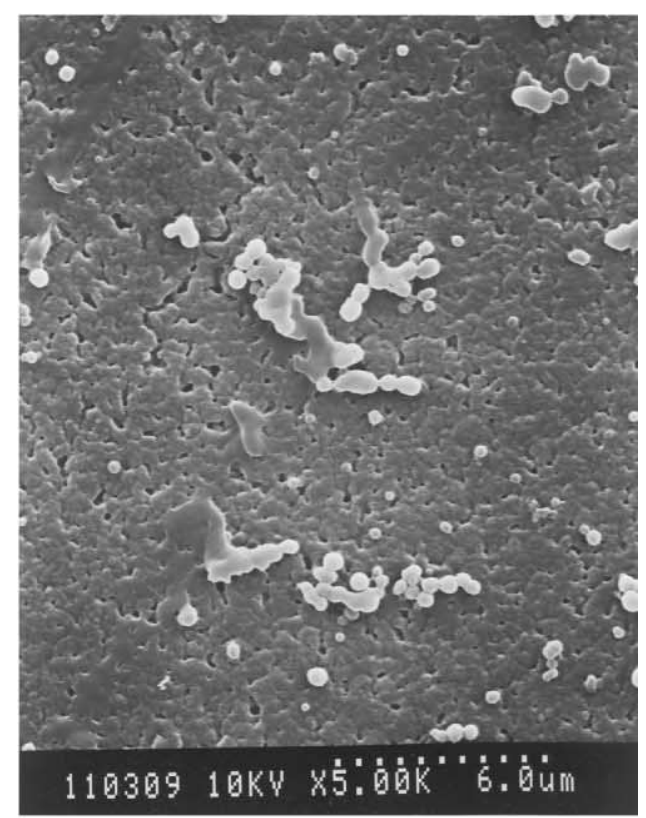

(a)

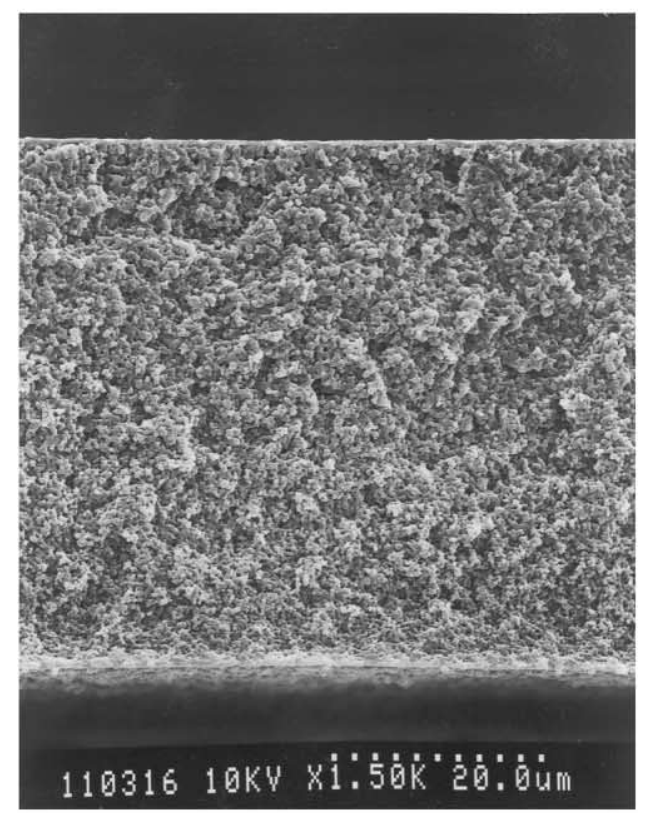

(b)

Fig. 1. SEM photographs of the R-1 membrane: (a) top surface; (b) cross-section.

for porosity on the surfaces between these two membranes used for cell culture test.

\subsection{Drug release study}

The releasing gradient of doxorubicin through the R-2 membrane was shown in Fig. 3. Accumulation of released doxorubicin was increasing for the first $72 \mathrm{~h}$. We believed that this was because that the EVAL particles on the

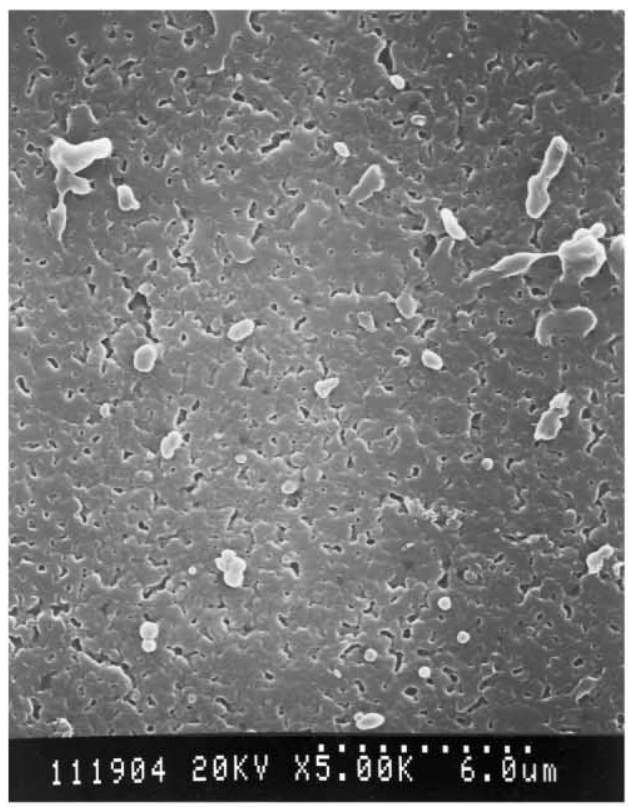

(a)

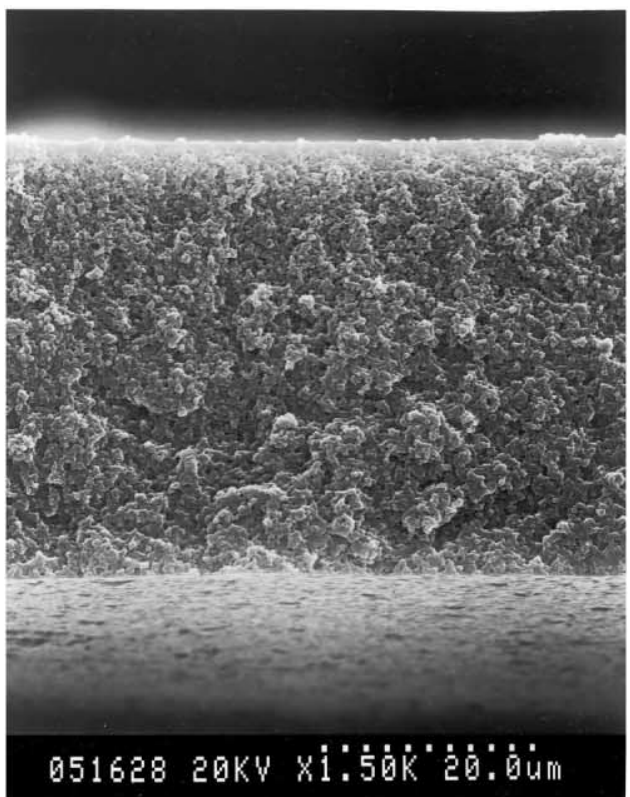

(b)

Fig. 2. SEM photographs of the R-2 membrane: (a) top surface; (b) cross-section

membrane surface did not coalesce completely. Therefore, doxorubicin could easily penetrate through the membrane and diffuse into the medium. Another finding is that a plateau-releasing pattern of doxorubicin was seen at the point of $2 \mathrm{~h}$ of experimental period and this phenomenon lasted for nearly $3 \mathrm{~h}$. If this release experiment was stopped at this point, most people could believe that doxorubicin was released in a simple and steady manner. However, another significant increase in drug delivery took place after the plateau-releasing stage and lasted for the next $68 \mathrm{~h}$. 


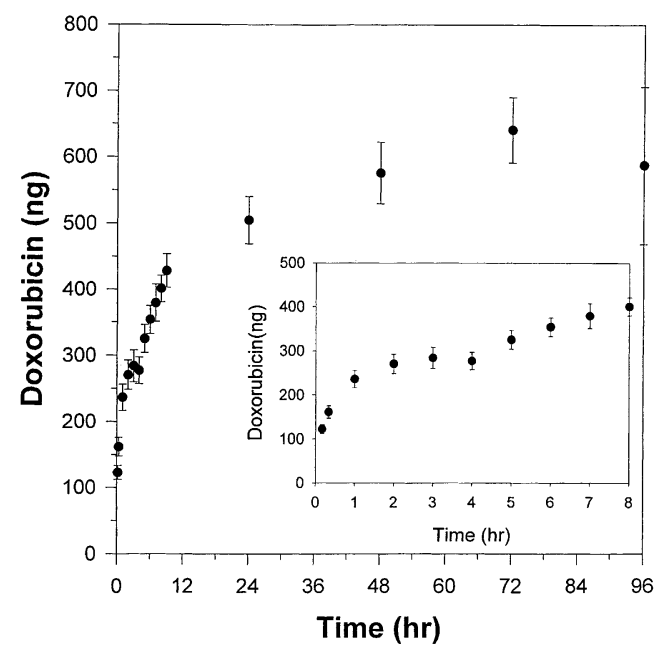

Fig. 3. Releasing gradient of doxorubicin through the EVAL membrane.

The total release amount of doxorubicin was approximately $640 \mathrm{ng}$ over the $72 \mathrm{~h}$ of experimental period.

Based on these results, a biphasic releasing pattern was noted for the doxorubicin, which could be probably due to non-homogeneity of drug distribution in the membrane. The first phase of drug release was a rapid response, which is assumed that some of the loaded drugs were accumulated in the voids among EVAL particles on the membrane surfaces. In comparison, the second phase was slow and prolonged which is probably due to the fact that drugs were encapsulated inside the EVAL particles as preparing the membranes. Therefore, the first phase of drug release was a rapid but transient response. With continuous exposure to medium, this first response phase faded, later to be followed by a slower and prolonged second phase. All these results demonstrated that the R-2 membrane could release the doxorubicin continuously at least for $72 \mathrm{~h}$.

\subsection{Cancer cell culture}

MTT reagent is a pale yellow substrate which can produce a dark blue formazan product as incubated with viable cancer cells. Therefore, the formazan reduced from the MTT is a useful index to reflect the level of cell metabolism. Fig. 4 shows the time course of formazan accumulation (percentage of control) for R-1 and R-2 membranes, whereas the control group was the tissue culture polystyrene dish with a silicon ring only. Formazan absorbance seen in this figure was an indication of the cancer cells seeded onto the R-1 and R-2 membranes, which were able to convert the MTT into a blue formazan product. It was found that the cell number on the R-1 membrane was significantly higher than that on the R-2 membrane $(P<0.01)$ at the point of 24,48 and $96 \mathrm{~h}$. Approximately $70-90 \%$ of cells relatively to the control adhered on the R-1 membrane. In comparison, the number of cancer cells on the R-2 membrane was decreasing with time where only $10-20 \%$ of cells relatively to the

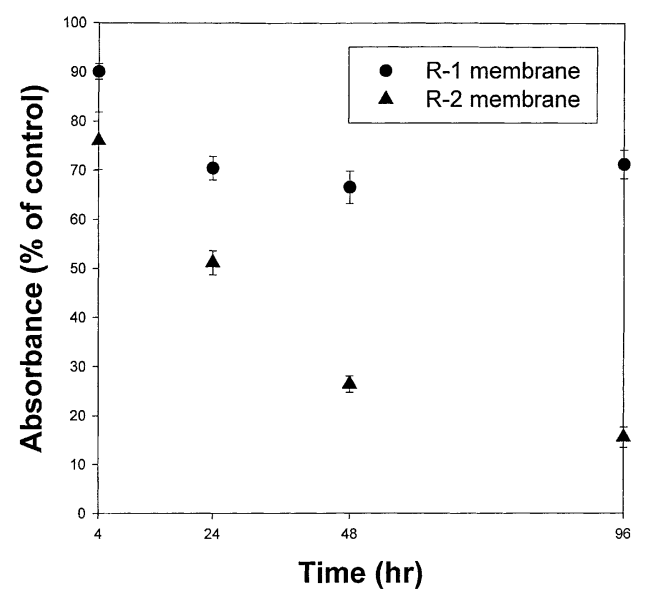

Fig. 4. MTT-tetrazolium assay for EVAL R-1 and R-2 membranes.

control were detected on the R-2 membrane after $96 \mathrm{~h}$ of experimental period. This result suggested that the growth of cancer cells was inhibited by the released doxorubicin from the R-2 membrane.

In conclusion, the EVAL is an ideal polymer matrix for the purpose of drug delivery, which can control the drug release in a two-step pattern. In addition, the doxorubicin encapsulated in the EVAL membrane can decrease the viability of cultured cancer cells.

\section{References}

[1] D.D. Lasic, Doxorubicin in sterically stabilized liposomes, Nature 380 (1996) 561-562.

[2] Y.W. Chien, Novel drug delivery systems, Marcel Dekker, New York, 1992.

[3] M.M. Gaspar, D. Blanco, M.E.M. Cruz, M.J. Alonso, Formulation of 1-asparaginase-loaded poly(lactide-co-glycolide) nanoparticles: influence of polymer properties on enzyme loading, activity and in vitro release, J. Controlled Release 52 (1998) 53-62.

[4] P. Giunchedi, H.O. Alpar, U. Conte, PDLLA microspheres containing steroids: spray-drying, o/w and w/o/w emulsifications as preparation methods, J. Microencapsulation 15 (1998) 185-195.

[5] W.L. Webber, F. Lago, C. Thanos, E. Mathiowitz, Characterization of soluble, salt-loaded, degradable PLGA films and their release of tetracycline, J. Biomed. Mater. Res. 41 (1998) 18-29.

[6] S. Loeb, S. Sourirajan, Sea water demineralization by means of an osmotic membrane., Adv. Chem. Ser. 38 (1963) 117.

[7] S. Yamashita, S. Nagata Takakura K, US Patent 4, 134,837,1979

[8] D.T. Lin, L.P. Cheng, Y.J. Kang, L.W. Chen, T.H. Young, Effects of precipitation condition on the membrane morphology and permeation characteristics, J. Membr. Sci. 140 (1998) 185-194.

[9] L.P. Cheng, H.Y. Lin, L.W. Chen, T.H. Young, Solute rejection of dextran by EVAL membranes with asymmetric and particulate morphologies, Polymer 39 (1998) 2135-2142.

[10] T.H. Young, W.Y. Chuang, C.W. Wei, C.Y. Tang, Investigation of the drug distribution and release characteristics from particulate membranes, J. Biomed. Mater. Res., 1999, submitted for publication.

[11] R. Duncan, F. Spreafico, Polymer conjugates: Pharmacokinetic considerations for design and development, Clin. Pharmacokinetic 27 (1994) 290-306.

[12] I.F. Uchegbu, J.A. Double, L.R. Kelland, J.A. Turton, A.T. Florence, The activity of doxorubicin niosomes against an ovarian cancer cell 
line and three in vivo mouse tumour models, J. Drug Targeting 3 (1996) 399-409.

[13] W.F. Fong, W. Lam, M. Yang, J.T.-F. Wong, Partial synergism between dextran-conjugated doxorubicin and cancer drugs on the killing of multidrug resistant KB-V1 cells, Anticancer Res. 16 (1996) 3773-3778.

[14] T.H. Young, J.Y. Lai, W.M. You, L.P. Cheng, Equilibrium phase behavior of the membrane forming water-DMSO-EVAL copolymer system, J. Membr. Sci 128 (1997) 55-65.
[15] R.E. Kesting, Synthetic Polymeric Membranes, Wiley, New York, 1985

[16] D.T. King, T.G. Venkateshwaran, J.T. Stewart, HPLC determination of a vincristine, doxorubicin, and ondansetron mixture in 0.9\% sodium chloride injection, J. Liquid Chromatogr. 17 (1994) $1399-1411$.

[17] T. Mosmann, Rapid colorimetric assay for cellular growth and survial: application to proliferation and cytotoxicity assays, J. Immunol. Methods 65 (1983) 55-63. 